\title{
Intracranial Hemangiopericytoma Focally Recurrent to the Pelvis
}

\author{
Robert Eil*, Kim C. Lu, George R. Wettach, Vassiliki L. Tsikitis
}

Department of Surgery, Oregon Health \& Science University, Portland, USA.

Email: *eil@ohsu.edu

Received June 20 ${ }^{\text {th }}$, 2012; revised July 25 ${ }^{\text {th }}$, 2012; accepted August $6^{\text {th }}, 2012$

\begin{abstract}
A 42-year-old male with a history of recurrent intracranial hemangiopericytoma presented to the Emergency Department with urinary retention. Ensuing work-up revealed a 10-centimeter circumscribed pelvic mass that appeared anatomically distinct from the sacrum. This metastasis was found 10 years after the initial resection of his intracranial lesion. An abdominal resection was performed, and the patient recovered uneventfully. Final pathology and staining findings were consistent with metastatic hemangiopericytoma. Hemangiopericytoma is a rare mesenchymal soft tissue neoplasm with potential for late local recurrence and metastasis. Here, we review the case and discuss the clinical relevance of narrowing the diagnosis to HPC or the growing umbrella of Solitary Fibrous Tumor (SFT). To our knowledge, this is the first report of intracranial HPC metastatic to the pelvis.
\end{abstract}

Keywords: Hemangiopericytoma; Solitary Fibrous Tumor; Intracranial; Immunohistochemical; Metastatic

\section{Introduction}

Sout and Murray originally described hemangiopericytoma (HPC) as a distinct entity in 1942. The medical community has long presumed that HPC originates from the pericytes of Zimmeran [1]. Therefore, HPC can occur anywhere throughout the body, but most frequently occurs in the lower extremities, pelvic fossa, and retroperitoneum [2]. Accounting for only $0.2 \%$ of intracranial neoplasms, intracranial HPC remains a rare event.

Small series and case reports have documented the ability of HPC to recur locally or metastasize after long intervals. Recurrence rates at 10 and 15 years have been documented with approximately $33 \%$ and $64 \%$ of patients, respectively. The most common sites of distant recurrence include bone and lung [3].

Here, we present an unusual case of intracranial HPC, recurring locally early on and finally metastasizing to the perirectal tissue, 10 years after initial resection.

\section{Case Presentation}

In 2003, this 36-year-old male, with a known history of previously resected occipital lobe intracranial hemangiopericytoma, presented with an intense headache, a dilated, fixed right pupil, and emesis. A head CT revealed a large right-sided intraparenchymal bleed (Figure 1) with resultant midline shift.

\footnotetext{
"Corresponding author.
}

An emergent decompressive craniotomy, hematoma evacuation, and re-resection of locally recurrent HPC were performed. The final pathology of the resected occipital specimen found recurrent/residual hemangiopericytoma.

The tumor had focal areas of necrosis, abundant mitotic figures, and was highly cellular with irregular, angular, hyperchromatic nuclei and sparse cytoplasm. Ad-

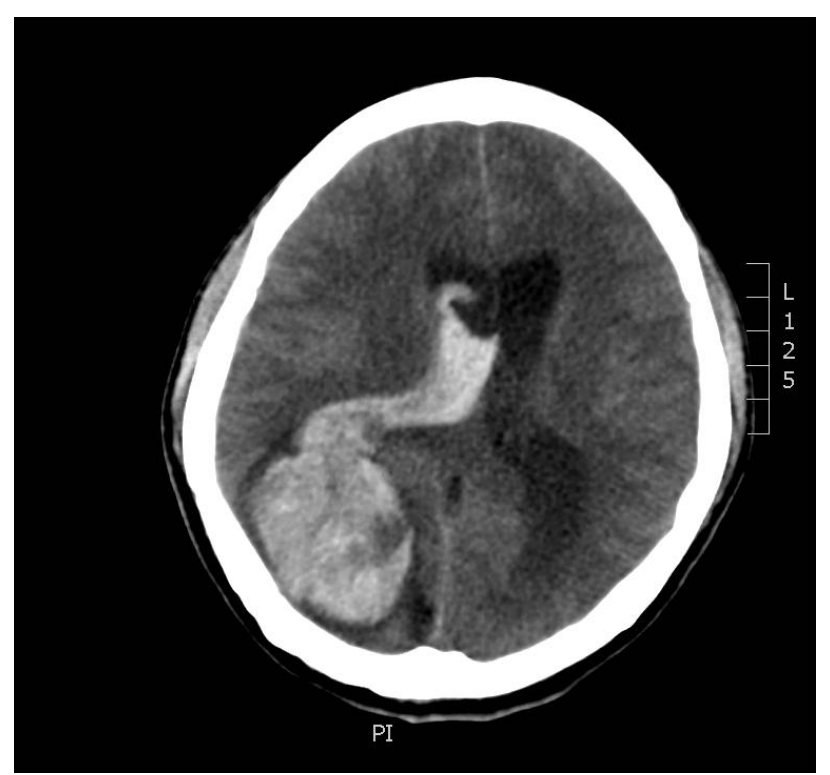

Figure 1. Large right-sided intraparenchymal bleed. 
ditionally, there were abundant thin-walled vessels with distinct staghorn configuration. Immunohistochemical (IHC) staining was negative for S-100 and EMA, but was positive for CD34.

The patient's postoperative course from his craniotomy was uneventful. He received a course of $54 \mathrm{~Gy}$ of radiation directed to the right occipital region later that year. In 2009, on a follow up MRI, the patient was noted to have a local intracranial recurrence and was treated with an additional $30 \mathrm{~Gy}$ of radiation to the right occipital lobe in the area of recurrence. He continued to receive routine follow up magnetic resonance imaging of his brain.

\subsection{Metastatic Presentation}

In December of 2011, at age 44, the patient presented acutely with urinary retention. Follow up CT and MRI imaging revealed an $8.5 \times 10 \mathrm{~cm}$ pelvic retroprostatic mass with heterogeneous attenuation and mass-effect on the adjacent bladder, and rightward displacement of the rectosigmoid colon (Figures 2-4). Subsequently, the

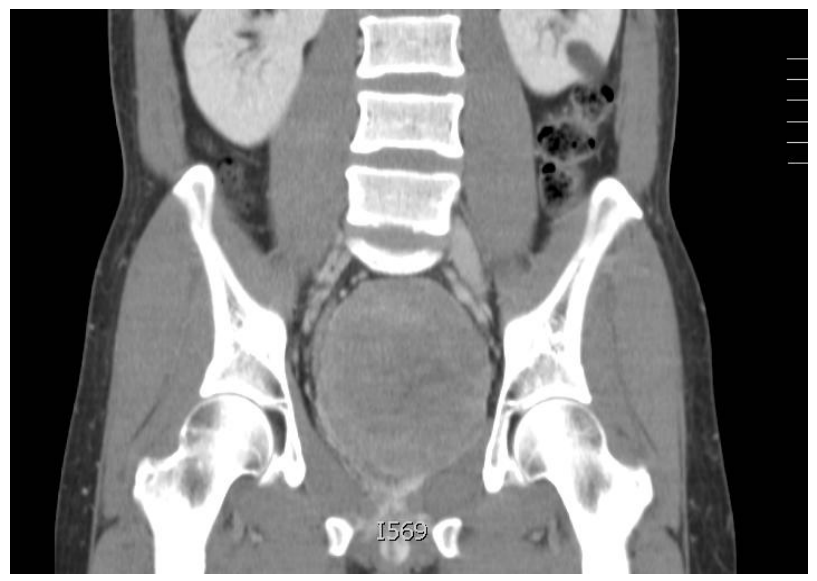

Figure 2. $8.5 \times 10 \mathrm{~cm}$ pelvic rectoprostatic mass.

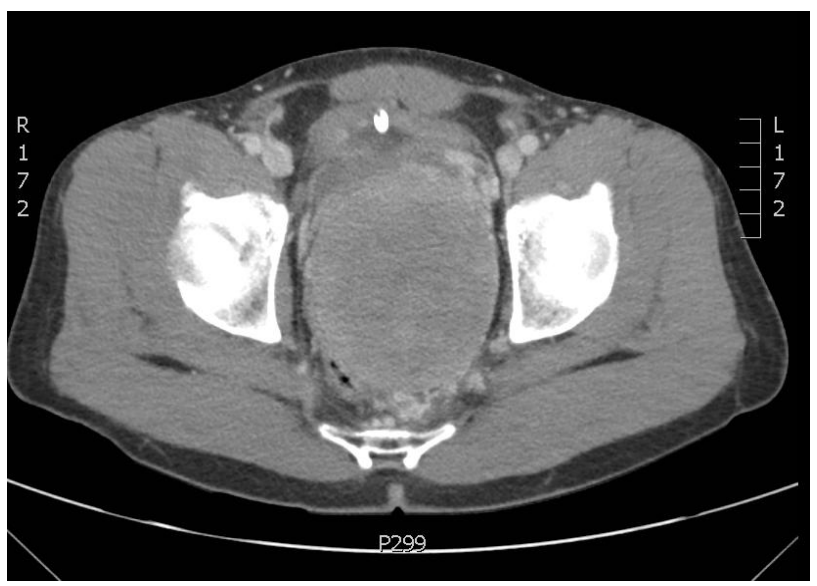

Figure 3. Heterogeneous attenuation and mass-effect on the adjacent bladder.

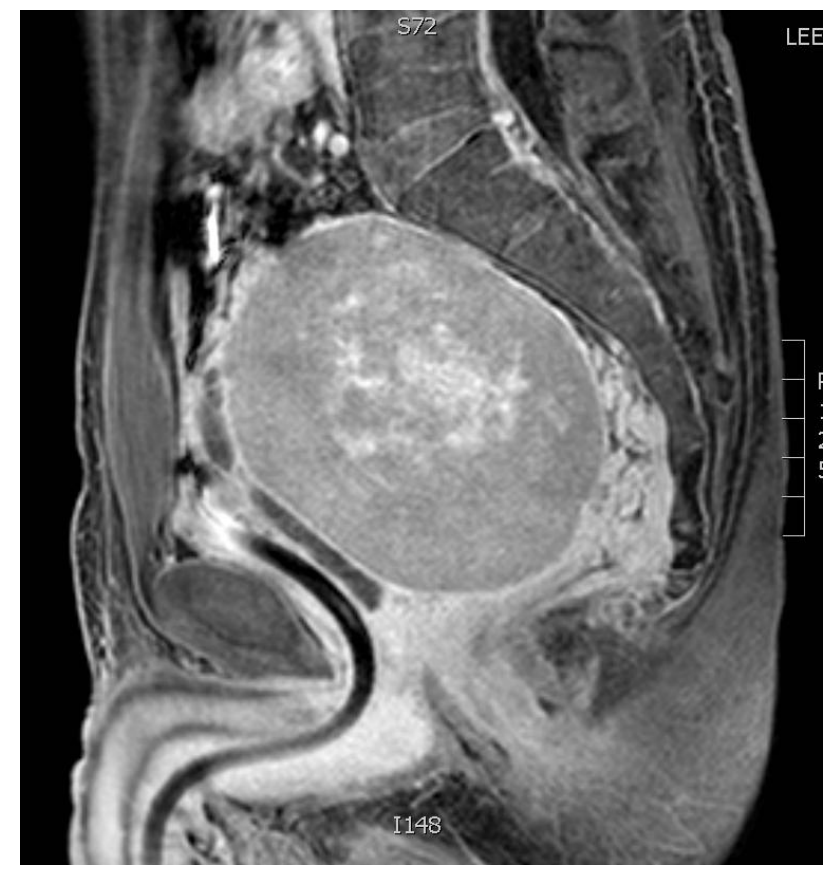

Figure 4. Rightward displacement of the rectosigmoid colon.

patient was taken to the operating room for laparotomy and an abdominal resection. Urologic consultants were unable to place ureteral stents, since they were unable to visualize the ureteral orifices during cystoscopy. The mass was found to be anterior and lateral to the rectum. It originated from Denonvilliers' fascia. Due to technical constraints, a step-wise low anterior resection with end colostomy was required. Initial frozen section interpretation found a spindle-cell pathology. Since the tumor was hypervascular, the blood loss was about 1.5 liters. The patient suffered no apparent perioperative complications and was discharged to home on post-operative day seven.

Given the finding of metastatic HPC and unknown margin status, an adjuvant course of radiation therapy was pursued. The patient received a total of 37.5 Gy to his pelvis. The patient is currently doing well and scheduled for continuing follow up and imaging surveillance.

\subsection{Pathologic Findings}

The sacral mass was composed of grossly soft to rubbery tissue, predominantly tan-pink in color with focal necrosis. It was adherent to the serosa of the rectum. Sections reveal numerous thin-walled and branching "staghorn" vessels surrounded by small ovoid to slightly fusiform cells with somewhat indistinct cytoplasmic borders (Figure 5). There was patchy CD-34 immunoreactivity (Figure 6), but CD-99, EMA, smooth muscle actin, beta-catenin, bcl-2, TLE-1, and S-100 stains were negative. Approximately twenty mitotic figures were present per ten high-power fields, and the proliferation index was be- 


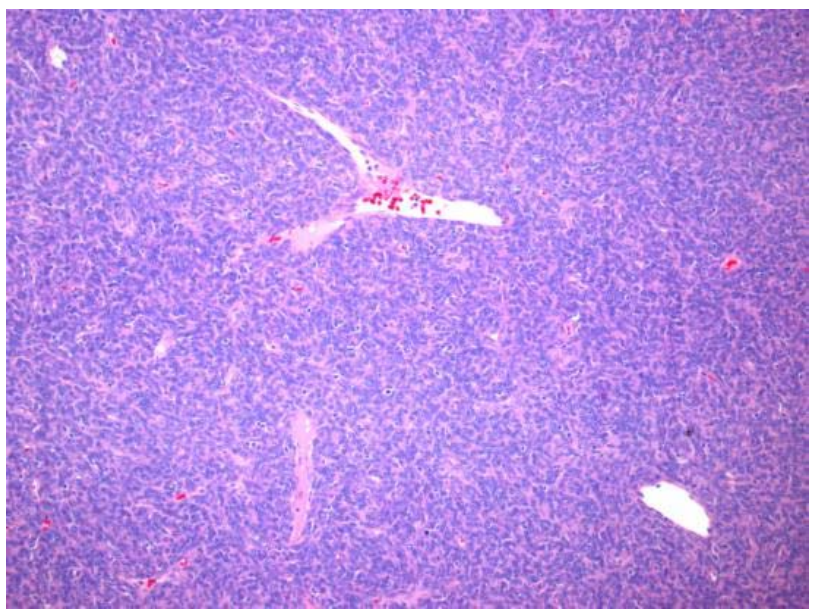

Figure 5. Numerous thin-walled, branching “staghorn” vessels are surrounded by small oval to slight fusiform cells with indistinct cell borders (Hematoxylin and eosin, 100×).

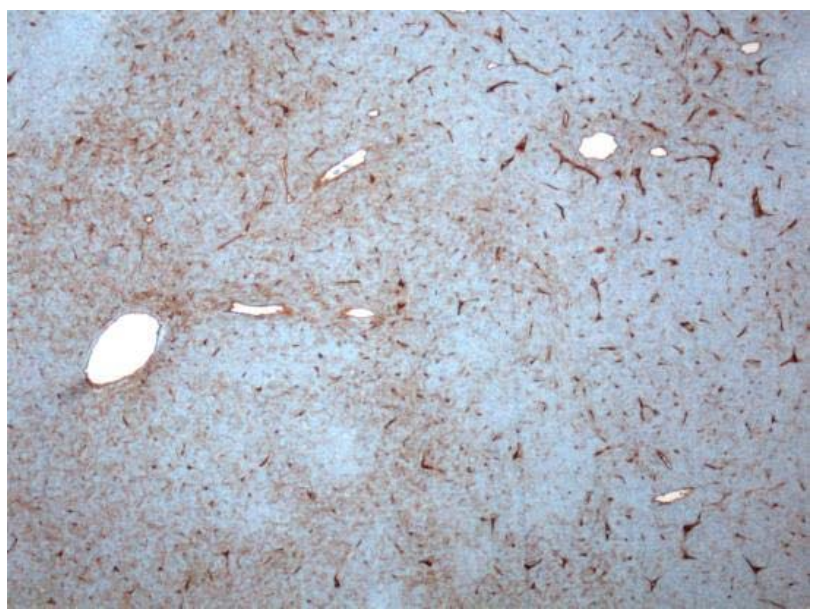

Figure 6. There is patchy CD34 immunoreactivity among the neoplastic cells, and the intervening vasculature serves as a positive internal control (CD34 IHC, 50×).

tween $10 \%$ and $20 \%$ by Ki-67 (Figure 7). Areas of necrosis were also readily apparent (Figure 8). These findings were consistent with malignant hemangiopericytoma (HPC).

\section{Discussion}

In his initial report in 1942, Stout described nine tumors as belonging to a new class of neoplasm, hemangiopericytoma [4,5]. Even within this initial cohort of nine, there were four separate varieties. In 1949, Stout examined an additional 25 cases of HPC, but again noted significant variability among these tumors in their size, shape, and intercellular connective tissue [6]. Ultimately, in 1953, Stout conceded that HPC could even be viewed as a diagnosis of exclusion [4]. With routine pathology not including immunohistochemistry until the 1980's and with HPC and solitary fibrous tumor (SFT) being virtually

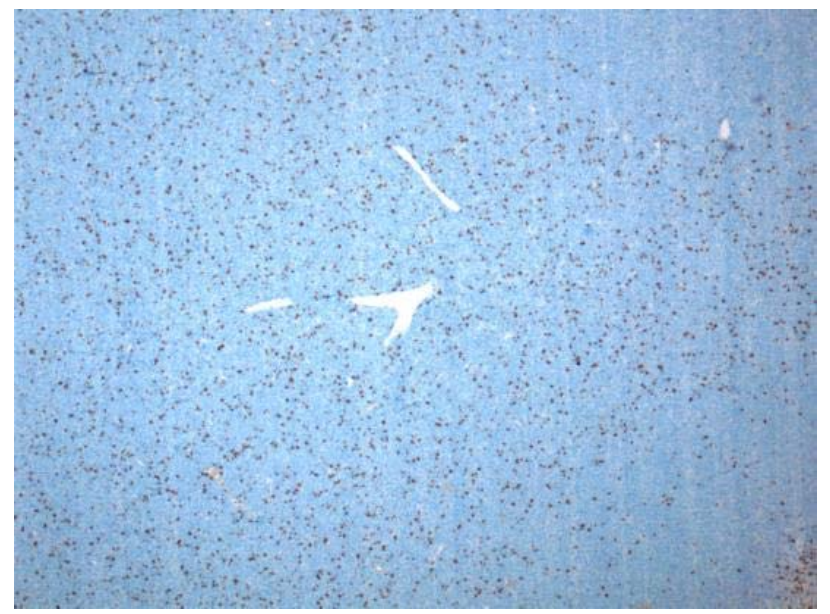

Figure 7. Within H\&E stained sections, approximately twenty mitotic figures are present per ten high power fields (not shown). A proliferation index of between $10 \%-20 \%$ is highlighted by Ki-67 immunostain in this low- magnification view (Ki-67 IHC, 50×).

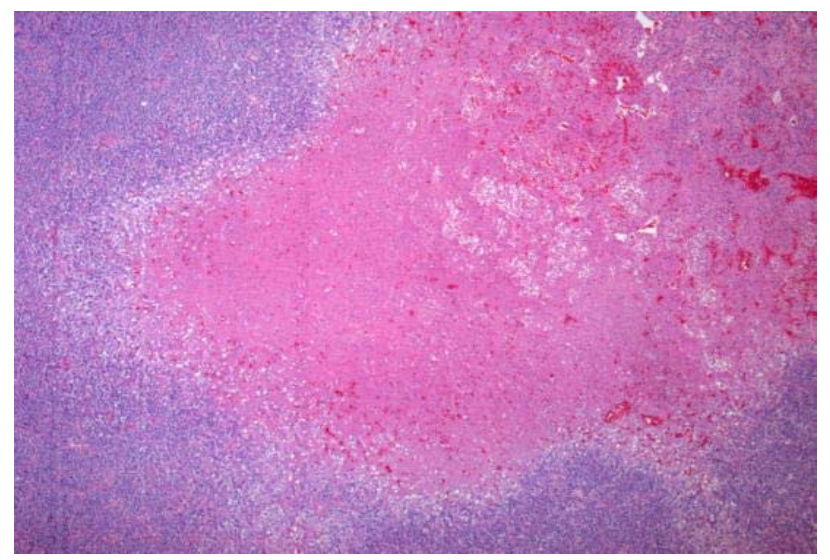

Figure 8. Several areas of necrosis are apparent at low magnification (Hematoxylin and eosin, 50×).

indistinguishable on light and electron microscopy, it is possible that previously assumed clinical characteristics of HPC have been a mischaracterization.

SFT was first described in 1870 as a neoplasm, typically of the pleura and of mesothelial origin. This was finally considered a unique entity in 1931 by Klemperer and Robin. Following recognition that SFT's could originate anywhere in the body, the number reported increased exponentially in the 1990's. Many tumors have progressively escaped from the broad definition of HPC over the past 10 years.

HPC and SFT are currently considered similar, if not identical neoplasms occupying ends of a morphologic spectrum [7]. The traditionally defined HPC is more cellular, with more rounded cells, and has a pericytic vascular arrangement. An SFT, by contrast, has more spindle-shaped cells and a more variable cellular arrangement, including the "patternless pattern". Although "antler” or 
"staghorn" vessels are present in both HPC and SFT, increased hyalinization is suggestive of SFT. While a small subset of HPC's express CD34, as they did in our case, SFT's are more consistently positive and would be immunoreactive for some combination of CD99, bcl-2, EMA, and actin [8,9]. Prior prognostic estimates of recurrence and survival likely included a mix of both low and high malignant potential lesions.

Most HPC's are benign, but criteria for malignancy, originally proposed by Enzinger and Smith [10], include large size $(>5 \mathrm{~cm}$ ), increased mitotic rate (greater than or equal to 4 mitoses per 10 high-power fields), and the presence of necrosis or hemorrhage. All three of these features were present in this case.

Very similar criteria have been applied more recently to malignant SFT neoplasms [11]. Given that both individual cellular SFT and HPC have variable clinical phenotypes, perhaps a solely morphologic characterization will allow appropriate studies of prognosis, benefit from adjuvant modalities, and need for regional and/or systemic surveillance.

With regard to this case presentation, the patient's intracranial neoplasm did show areas of necrosis and dense mitotic figures. Aggressive locoregional surveillance with MRI's every 6 months and therapy with radiation therapy was pursued; the patient, however, had a distant recurrence. HPC can infrequently recur at remote sites from its primary lesion. The distribution of metastases is varied and unpredictable, with the most common sites for intracranial HPC being bone, liver, lung, CNS, and abdominal cavity in decreasing order [3]. Thus, surveillance for metastasis would be challenging and difficulty to tailor.

Sites of metastasis are varied and difficult to predict; this is the first report of intracranial HPC metastatic to the pelvis. Ultimately, applying the weighted criteria originally proposed by Enzinger in 1976 may be the most valuable in this case: any soft tissue neoplasm with focal areas of necrosis and packed with mitotic figures will have an aggressive pattern of behavior and should be treated as such.

\section{Acknowledgements}

The authors would like to thank Mary Kwakosky-Lawlor for her assistance in the editing and preparation of the bibliography of this review.

\section{REFERENCES}

[1] C. Gengler and L. Guillou, "Solitary Fibrous Tumour and
Haemangiopericytoma: Evolution of a Concept," Histopathology, Vol. 188, No. 5, 2006, pp. 63-74. doi:10.1111/j.1365-2559.2005.02290.x

[2] F. R. Spitz, M. Bouvet, P. W. Pisters, R. E. Pollock and B. W. Feig, "Hemangiopericytoma: A 20-Year Single-Institution Experience,” Annals of Surgical Oncology, Vol. 5, No. 4, 1998, pp. 350-355. doi:10.1007/BF02303499

[3] H. Suzuki, Y. Haga, K. Oguro, S. Shinoda, T. Masuzawa and N. Kanai, "Intracranial Hemangiopericytoma with Extracranial Metastasis Occurring after 22 Years,” Neurologia Medico-Chirurgica, Vol. 42, No. 7, 2002, pp. 297- 300. doi:10.2176/nmc.42.2977

[4] A. P. Stout, "Case 5," Tumour Seminar of the Arthur Purdy Stout Club, New York, 6 June 1953.

[5] A. P. Stout and M. R. Murray, "Hemangiopericytoma: A Vascular Tumor Featuring Zimmermann's Pericytes,” Annals of Surgery, Vol. 116, No. 1, 1942, pp. 26-33. doi:10.1097/00000658-194207000-00004

[6] A. P. Stout, "Hemangiopericytoma: A Study of TwentyFive New Cases,” Cancer, Vol. 2, No. 6, 1949, pp. 10271035.

doi:10.1002/1097-0142(194911)2:6<1027::AID-CNCR28 20020609>3.0.CO;2-R

[7] L. Guillou, J. A. Fletcher, C. D. Fletcher and N. Mandahl, "Extrapleural Solitary Fibrous Tumor and Hemangiopericytoma," In: C. D. Fletcher, K. K. Unni and F. Mertens, Eds., World Health Organization Classification of Tumours: Pathology and Genetics of Tumours of Soft Tissue and Bone. Pathology, IARC Press, Lyon, 2002, pp. 86-90.

[8] S. Suster, A. G. Nascimento, M. Miettinen, J. Z. Sickel and C. A. Moran, "Solitary Fibrous Tumors of Soft Tissue. A Clinicopathologic and Immunohistochemical Study of 12 Cases,” The American Journal of Surgical Pathology, Vol. 19, No. 11, 1995, pp. 1257-1266. doi:10.1097/00000478-199511000-00005

[9] M. Chilosi, F. Facchettti, A. P. Dei Tos, M. Lestani, M. L. Morassi, G. Martignoni, et al., "Bcl-2 Expression in Pleural and Extrapleural Solitary Fibrous Tumours," The Journal of Pathology, Vol. 181, No. 4, 1997, pp. 362-367. doi:10.1002/(SICI)1096-9896(199704)181:4<362::AID-P ATH764>3.0.CO;2-Y

[10] F. M. Enzinger and B. H. Smith, "Hemangiopericytoma: An Analysis of 106 Cases,” Human Pathology, Vol. 7, No. 1, 1976, pp. 61-82. doi:10.1016/S0046-8177(76)80006-8

[11] A. V. Vallat-Decouvelaere, S. M. Dry and C. D. Fletcher, "Atypical and Malignant Solitary Fibrous Tumors in Extrathoracic Locations: Evidence of Their Comparability to Intra-Thoracic Tumors," The American Journal of Surgical Pathology, Vol. 22, No. 12, 1998, pp. 1501-1511. doi:10.1097/00000478-199812000-00007 